\title{
A UNIVERSAL METHOD FOR EFFUSIVE-FLOW CHARACTERIZATION OF TARGET/VAPOR TRANSPORT SYSTEMS FOR RIB GENERATION
}

\author{
J. C. Bilheux ${ }^{1,2}$, G. D. Alton, Y. Liu, S. N. Murray, C. Williams and C. A. Reed. \\ Oak Ridge National Laboratory ${ }^{*}$, P.O. Box 2008, \\ Oak Ridge TN 37831-6368, USA.
}

\section{Abstract}

Worldwide interest in the use of accelerated radioactive ion beams (RIBs) for exploring reactions important in understanding the structure of the nucleus and nuclear astrophysical phenomena has motivated the construction of facilities dedicated to their production and acceleration. Many facilities utilize the Isotope-Separator-On-Line (ISOL) method in which species of interest are generated within a solid or liquid target matrix. Experimentally useful RIBs are often difficult to generate by this technique because of the times required for diffusion from the interior of the target material, and to effusively transport the species of interest to the ion source following diffusion release in relation to its lifetime. Therefore, these delay times must be minimized. We have developed an experimental method that can be used to determine effusive-flow times of arbitrary geometry target/vapor transport systems. The technique utilizes a fast valve to measure effusive-flow times as short as 0.1 $\mathrm{ms}$ for any chemically active or inactive species through any target system, independent of size, geometry and materials of construction. In this report, we describe the effusive-flow experimental arrangement and provide time spectra for noble gases through prototype RIB target/vapor-transport systems.

\section{INTRODUCTION}

The Isotope Separator On-Line (ISOL) technique is most frequently used to produce short-lived isotopes for research at radioactive ion beam (RIB) facilities such as the Holifield Radioactive Ion Beam Facility (HRIBF) [1]. After being created in the matrix of a solid or liquid target, the short-lived species must diffuse from the target material and then be transported in gaseous or vapor form through the transport system to an ion source where they are ionized and accelerated. Decay losses associated with the times required for the diffusion and effusive-flow processes to take place are principal means whereby short half-life radioactive species are lost between initial formation and utilization. Thus, it is desirable to minimize the times associated with both processes. An experimental method has been developed that can be used to determine effusive-flow times for arbitrary geometry target/vapor transport systems. In this report, we present effusive-flow time spectra experimentally measured for two different target/vapor transport systems with and without Reticulated Vitreous Carbon Foam (RVCF) target material. The aim of the present studies is to demonstrate the viability of the technique with the ultimate objective of optimally coupling HRIBF targets to existing ion sources and to develop a technique that can be universally used to characterize present and future target/vapor transport systems at ISOL facilities.

\section{EFFUSIVE-FLOW FORMULA}

For an ideal gas in a tube of radius, $a$, and length, $l$, at low pressure, the steady-state flow rate, $d N / d t$, for particles of average velocity, $v$, flowing through a tube under a density gradient along the tube, $d n / d z$, is given by [2]:

$$
\mathrm{dN} / \mathrm{dt}=-\left\{2 \mathrm{n} \pi^{3} / 3\right\} \vee \mathrm{dn} / \mathrm{dz}=-\left\{2 \mathrm{n} \pi^{3} / 3 \mathrm{k}_{\mathrm{B}} \mathrm{T}\right\} \nu \mathrm{dp} / \mathrm{dz}
$$

where $n$ is the particle density, $v$ is the Maxwellian velocity and $k_{B}$ is Boltzmann's constant.

The average transport time for chemically inactive radioactive particles with lifetime, $\tau_{1 / 2}$, to flow through a tubular system can be deduced by solving the time dependent form of Eq.1. The resulting equation is:

$$
\mathrm{N}=\mathrm{N}_{0} \exp [-\lambda \mathrm{t}] \exp \left[-\mathrm{t} / \tau_{\mathrm{c}}\right]
$$

where $N_{0}$ is the number of particles in the volume at time $t=0 ; \lambda=0.693 / \tau_{1 / 2} ;$ the characteristic time, $\tau_{\mathrm{c}}$, is given by $\tau_{\mathrm{c}}=3 / 4\left[\mathrm{~N}_{\mathrm{b}} \tau_{0} \exp \left(-\mathrm{H}_{\mathrm{a}} / \mathrm{k}_{\mathrm{B}} \mathrm{T}\right)+\mathrm{L} / \mathrm{v}\right]$ where $v=\left(8 \mathrm{k}_{\mathrm{B}} \mathrm{T} / \pi \mathrm{M}\right)^{1 / 2}$; $H_{a d}$ is the enthalpy of adsorption; and $\tau_{0}=3.4 \cdot 10^{-15} \mathrm{~s}$.

\section{EXPERIMENTAL ARRANGEMENT}

The fast closing valve system (Fig. 1) consists of an electro-pneumatic actuator [3] that drives a shutter for closing gas flow to the target reservoir. The fast closing valve system, initially designed to close in $10 \mathrm{~ms}$, has been modified in order to achieve a closing time of 0.1 ms. Effusive flow times as short as $0.1 \mathrm{~ms}$ can then be measured. To experimentally measure time spectra, the fast closing valve system is close-coupled to the target material reservoir in order to minimize contributions

\footnotetext{
${ }^{1}$ bilheux@mail.phy.ornl.gov

${ }^{2}$ Ph.D. Graduate Student, Université de Versailles-Saint-Quentin, Versailles, France.

* Managed by UT-Battelle, LLC, for the U.S. Department of Energy under contract DE-AC05-00OR22725
} 


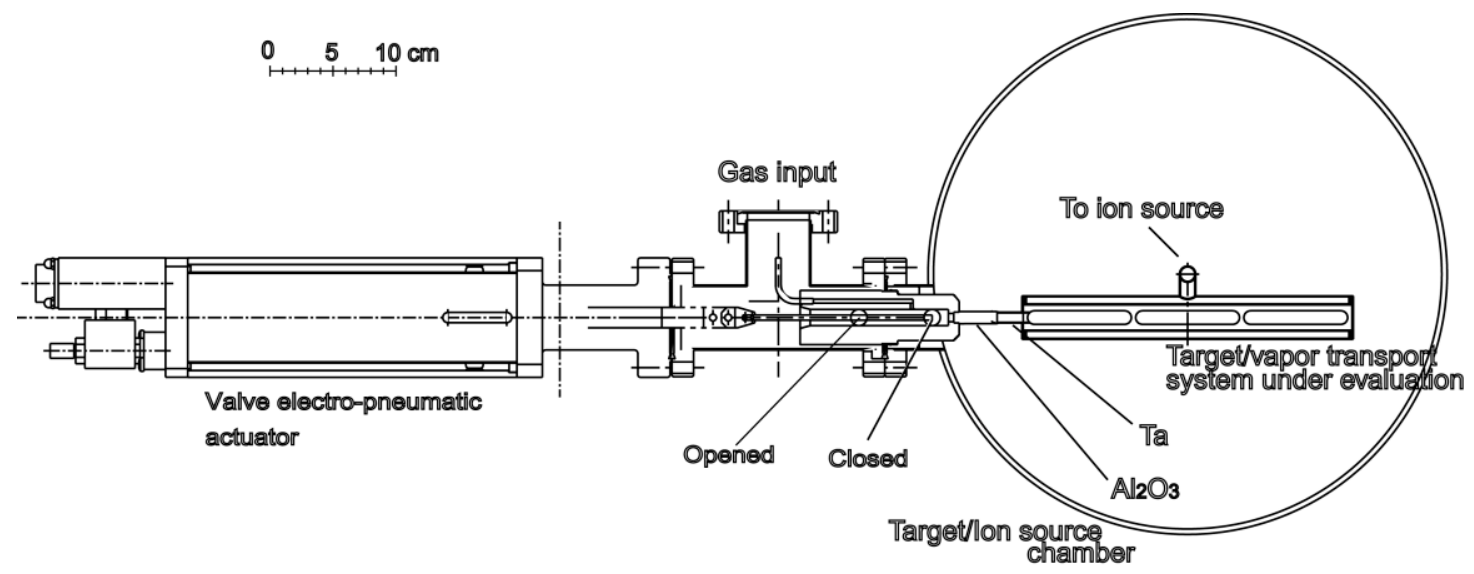

Figure 1: Schematic drawing of the fast closing valve, gas input system on the target/ion source chamber.

to the delay function which result from particle hold-up in the injection feed-line. The gas of interest is injected via the gas input through the target/vapor transport system to the Electron Beam Plasma Ion Source (EBPIS) [4] where it is ionized and accelerated. The mean-effusive delay time is determined by rapidly interrupting flow into the Target Ion Source (TIS) while monitoring the time dependence of the mass selected ion current. The target reservoir is resistively heated by passing current through a heater that surrounds the target. The temperature of the target reservoir is changed by varying the electrical current flowing through the target heater. Its magnitude is measured with an optical pyrometer as well as a thermocouple. $\mathrm{An} \mathrm{Al}_{2} \mathrm{O}_{3}$ tube is used to connect the electro-pneumatic actuator to the target/vapor transport system under evaluation in order to electrically isolate the fast valve from the target reservoir.

\section{EFFUSIVE DELAY-TIME MEASUREMENTS}

Time spectra were measured for noble gases flowing through two different target reservoir/transport systems to an electron Beam Plasma Ion source (EBPIS), with and without Reticulated Vitreous-Carbon Foam (RVCF) in each reservoir. The two target reservoir geometries are shown in Fig. 2. The target reservoirs are loaded with RVCF of the same diameter $(15 \mathrm{~mm})$ and length $(193 \mathrm{~mm})$. A $4.3 \mathrm{~mm}$ annular space is provided around the RVCF in the parallel-flow system shown in Fig. 2b. to increase the conductance of the reservoir. The radial direction of the RVCF holder is $58 \%$ open.

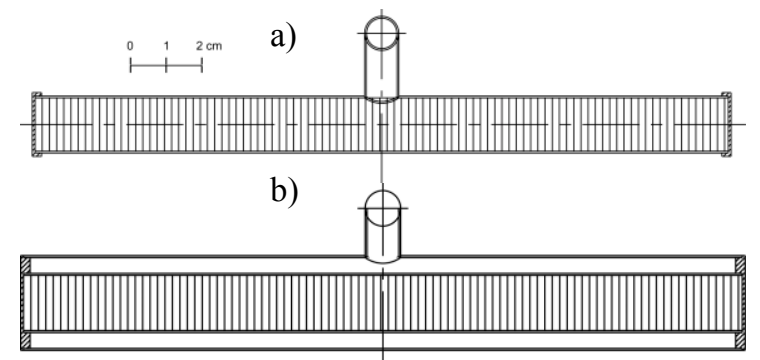

Figure 2: a) The serial-tlow and, b) parallel-tlow reservoirs to be characterized.

\subsection{Serial-coupled system}

The characteristic times for noble gas elements ( $\mathrm{He}, \mathrm{Ne}$, $\mathrm{Ar}, \mathrm{Kr}$ and $\mathrm{Xe}$ ) flowing through the serial coupled system with and without Reticulated Vitreous Carbon Foam are displayed in Fig. 3. for different temperatures.

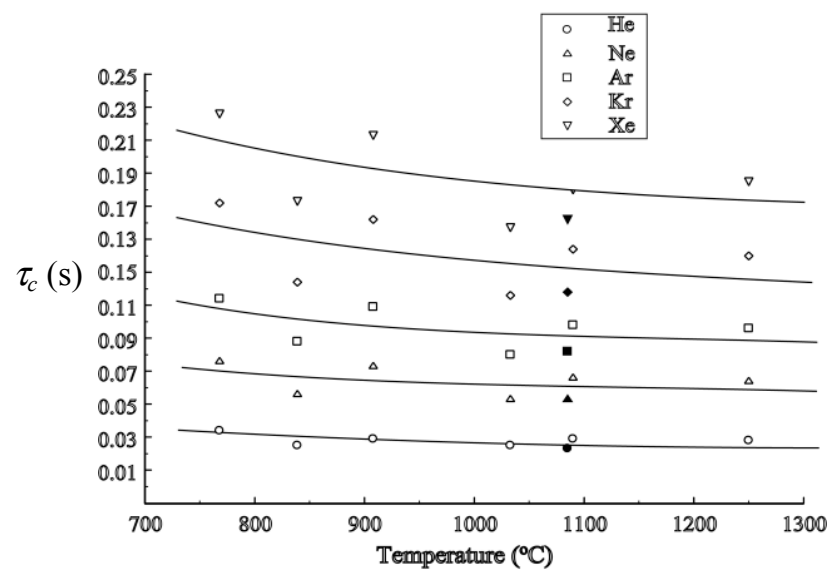

Figure 3: Characteristic times, $\tau_{c}$, for noble gases versus temperature, $T$, of the target material reservoir with (open symbols) and without RVCF material (solid symbols).

As expected, the characteristic times are shorter for lighter species. The decrease in characteristic time for noble gases with increasing temperature, $T$, are attributable to the fact that $\tau_{\mathrm{c}}$ is proportional to $T^{-1 / 2}$ (see Eq. 2 and the definition of $\tau_{c}$ ).

As noted, the effusion flow time of the slowest species studied (Xe) varies from $0.21 \mathrm{~s}$ at $\mathrm{T}=800{ }^{\circ} \mathrm{C}$ to $0.17 \mathrm{~s}$ at $\mathrm{T}=1300^{\circ} \mathrm{C}$. The RVCF does not significantly increase the effusive-flow times of the noble gases, thus, validating the importance of high permeability targets for RIB applications.

\subsection{Parallel-coupled system}

Fig. 4 shows the characteristic time for noble gases flowing through the parallel-coupled system with and without RVCF. As was the case for the serial coupled 
system, the effusive flow times are shorter at higher temperatures. As noted, the presence of RVCF slightly decreases the characteristic times for the gases in this configuration.

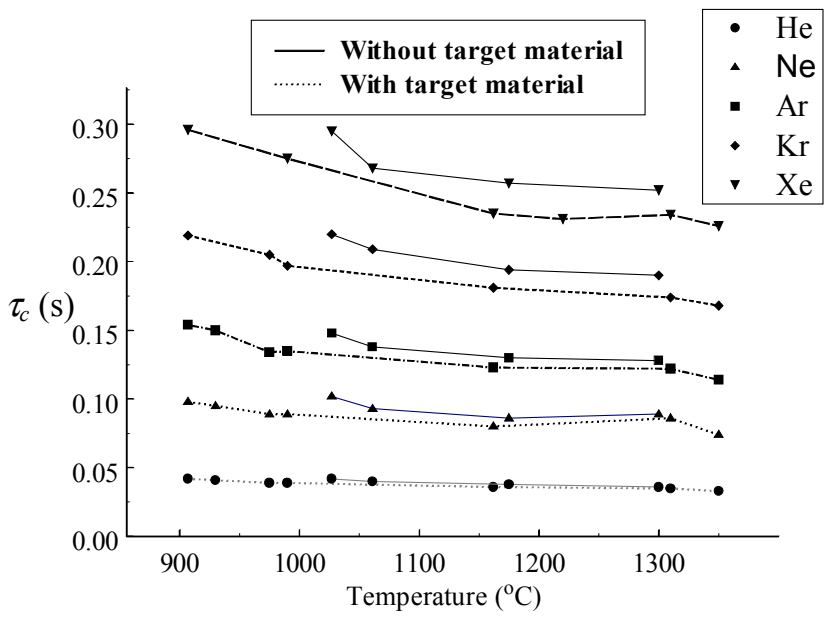

Figure 4: Characteristic time, $\tau_{\mathrm{c}}$, for noble gases versus target material reservoir temperature, $T$, with and without RVCF material.

\section{DISCUSSION}

For the same temperature, the effusive-flow times are longer for the parallel-coupled system than for the serialcoupled system. This can be explained because the average distance traveled per particle is longer for the larger diameter tube. These studies show that the RVCF does not significantly affect effusive flow times. For the parallel-flow configuration, the characteristic times are shortened by its presence.

Since the enthalpies of adsorption for the noble gases are essentially zero [5], the average distance traveled per particle, $L$, through the target/vapor transport system can be determined from Eq. 2. $L$ was found to have respective values of $97 \mathrm{~m}$ and $82 \mathrm{~m}$ with and without RVCF material in the case of the serial-coupled system and $134 \mathrm{~m}$ and $142 \mathrm{~m}$ for the parallel coupled system. Fig. 5 clearly shows that the characteristic effusive-flow times for noble gases are proportional to $M^{1 / 2}$. Thus, for these gases, the sticking times on the walls of the transport system and target surfaces are negligible.

\section{FUTURE STUDIES}

By using the time spectra of CO for which the enthalpy of adsorption is known [6], $\mathrm{Nb}$ can be determined. It will then be possible to completely characterize a given target/vapor transport system, independent of species and geometry, since the average distance traveled per particle, $L$, and the average number of bounces, $N_{b}$, are known. The ultimate objective of the investigations is to develop a technique for designing an optimum target/vapor transport system that minimizes effusive- flow to a given ion source. Fig. 6 displays a high vacuum conductivity scenario for potential use at the HRIBF.

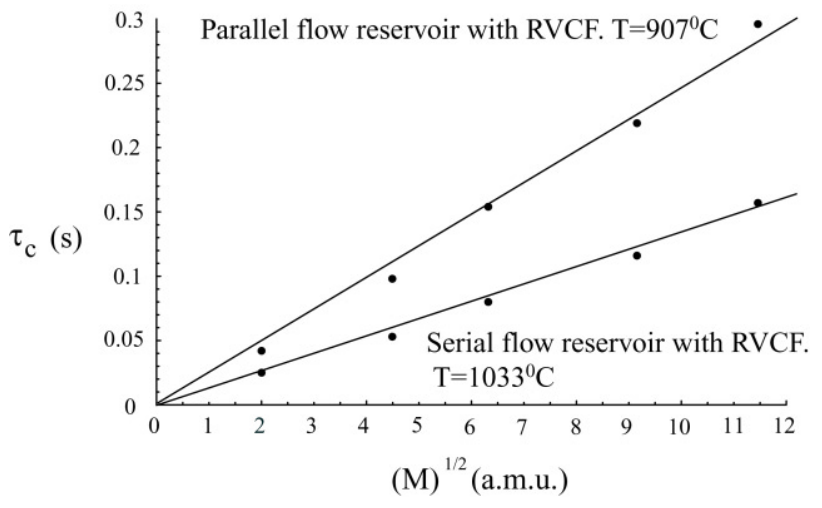

Figure 5: Measured characteristic effusive-flow times, $\tau_{\mathrm{c}}$, for noble gases versus $(M)^{1 / 2}$. Experiment: $\bullet$; Theory -

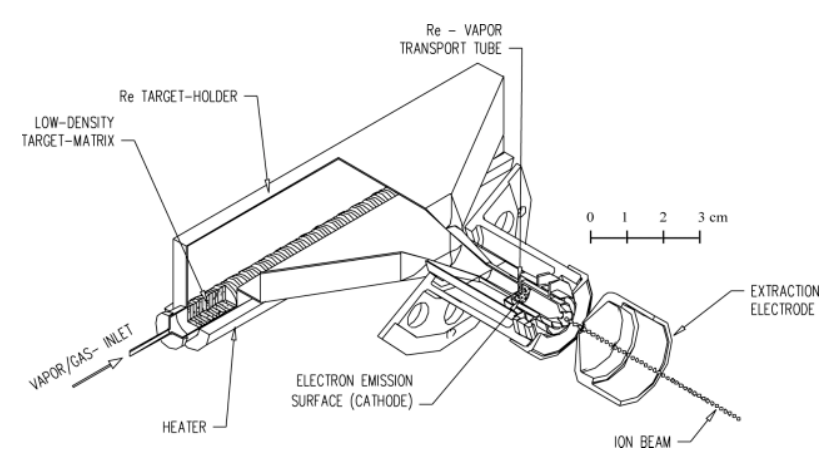

Figure 6: High vacuum conductivity target/vapor transport/ion source system for fast effusive-flow.

\section{REFERENCES}

[1] G. D. Alton, J. R. Beene, J. Phys. G; Nucl. Particle Phys., 24 (1998) 1347.

[2] R. D. Present, Kinetic Theory of Gases, Mc Graw-Hill Book Company (1958).

[3] VAT Vakuumventile AG, CH-9469 Haag, Switzerland.

[4] G. D. Alton, Nucl. Instr. and Meth. in Phys. Res. A 382 (1996) 207.

[5] H. J. Kreuzer and Z. W. Gortel, Physisorption Kinetics, Springer-Verlag, Berlin, 1986, p. 5.

[6] A. Zangwill, Physics at Surfaces, Cambridge University Press, Cambridge, 1988, p. 208. 